\title{
Characterizing the Radiation Fields in the Atmosphere Using a Cloud-Aerosol-Radiation Product From Integrated CERES, MODIS, CALIPSO and CloudSat Data
}

\author{
Patrick Minnis, Bruce Wielicki, Charles A. Trepte \\ NASA Langley Research Center \\ Hampton, VA USA \\ p.minnis@nasa.gov
}

Sunny Sun-Mack, Yan Chen, Sharon Gibson

SSAI

Hampton, VA USA

\author{
Seiji Kato \\ National Institute of Aerospace \\ Hampton, VA USA \\ Madison, WI USA \\ Graeme Stephens \\ Department of Atmospheric Science \\ Colorado State University \\ Ft. Collins, CO USA
}

\begin{abstract}
CloudSat and CALIPSO cloud and aerosol information is convolved with CERES and MODIS cloud and radiation data to produce a merged 3-dimensional cloud and radiation dataset.
\end{abstract}

Keywords-component; clouds; radiation; CALIPSO; CloudSat

\section{INTRODUCTION}

Understanding the relationships between atmospheric components and the radiation fields within and external to the atmosphere is critical for constraining weather and climate models and, possibly, for improving the same through assimilation of those fields. Our current understanding of those relationships and the means to estimate those radiation fields on a global basis are limited to a 2 or 2.5 dimensional characterization. Current passive remote sensing techniques using multispectral radiances measured by imagers, such as the MODerate-resolution Imaging Spectroradiometer (MODIS) on the Aqua satellite, can generally retrieve the bulk optical properties of a cloud system and provide an estimate of the cloud top and a cruder assessment of the cloud base for singlelayered clouds. In scenes free of clouds, it is possible to retrieve the bulk optical properties of the aerosol column from the same imagers. Broadband radiometers, such as those developed for the Clouds and the Earth's Radiant Energy System (CERES) [1], can be used to determine the total outgoing longwave and shortwave radiative fluxes, thereby, defining the amount of radiative energy available for heating the atmosphere and surface, or for evaporating water. Various algorithms are now available for using that same information to estimate the radiation budget at the surface, so that the amount of radiant energy deposited in the atmosphere can also be estimated.

By combining the passive retrievals from MODIS [2] with the broadband radiative fluxes, the CERES project has made substantial advances in characterizing the relationships between atmospheric components and the radiation budget. Yet, many questions and uncertainties remain. Clouds often occur in overlapping layers and, therefore, many of the derived cloud properties are often very inaccurate. For example, an ice cloud overlapping a water cloud is generally interpreted as an ice cloud and the resulting optical depths and water paths can be extremely biased. Aerosols can only be retrieved in clear skies and there is no knowledge of their vertical distribution. Thus, it is not possible to accurately place clouds and aerosols in the same layers and determine the indirect effect of the aerosols without many assumptions. Furthermore, the vertical impact of the radiative heating by the aerosols cannot be reliably characterized without knowledge of the aerosol altitudes. Overall, the vertical heating rate profile is very difficult to determine with any accuracy from passive satellite measurements because of these and other shortcomings. The A-Train, which includes Aqua, provides new resources for unscrambling the vertical profiles of clouds and aerosols while simultaneously measuring broadband fluxes.

Pulling the various A-Train datasets together to determine the vertical structure of the cloud and aerosol fields and their impact on the atmospheric radiation budget is a challenge due to the different fields of view and nature of the several datasets. This paper describes the integration of the passive MODIS and CERES measurements with the vertical profile information 
derived from the cloud radar on CloudSat [3] and from the lidar on the Cloud-Aerosol Lidar and Infrared Pathfinder Observation (CALIPSO) Satellite [4]. Methods to distribute the information from the small CALIPSO and CloudSat footprints into the larger MODIS and CERES fields of view are discussed. Initial comparisons of the passive and active fields are presented along with initial estimates of the heating rates. The combined datasets should provide an unprecedented characterization of the atmospheric heating rates as well as a better understanding of the impacts of clouds and aerosols on the radiation budget.

\section{DATA AND MATCHING}

\section{A. CERES}

The CERES-MODIS (CM) cloud analysis algorithms use the $1-\mathrm{km} 0.65,1.38,1.64$ (2.13), 3.8, 6.7, 8.5, 10.8, and 12.0 $\mu \mathrm{m}$ channels. The CERES scanners measure broadband shortwave, total, and infrared window $(8-12 \mu \mathrm{m})$ radiances with a nadir footprint of $\sim 20 \mathrm{~km}$. The CERES instrument scans from limb to limb, while the MODIS scans out to a $55^{\circ}$ scan angle that translates to a viewing zenith angle (VZA) of $\sim 70^{\circ}$. Thus, CERES data are analyzed out VZA $=70^{\circ}$. Each MODIS pixel is analyzed to retrieve cloud properties as described in [2] and the references therein. The results are then convolved into the CERES footprint to associate a given cloud-surface scene with a broadband radiance measurement that is then inverted with a scene-based anisotropic directional model to determine the top-of-atmosphere irradiance.

\section{B. CALIPSO and CloudSat}

CALIPSO and CloudSat are off the Aqua nadir by several degrees. The CERES field of view (fov) having a center point closest to the CALIPSO ground track is matched to a segment of the CALIPSO data stream. The CALIPSO lidar (CALIOP) takes a vertical profile every $333 \mathrm{~m}$ along track and has a $70-\mathrm{m}$ fov. It is aligned with the $125-\mathrm{m}$ resolution CALIPSO visible imager and every 3 or 4 CALIOP profiles are matched with the nearest $1-\mathrm{km}$ MODIS pixel. An idealized cloud and aerosol field is also indicated in Figure 1 with a clear portion, scattered cumuli between 1.5 and $3 \mathrm{~km}$, broken cirrus between 11 and 12 $\mathrm{km}$, and a uniform aerosol layer centered at $2 \mathrm{~km}$. The pattern of lidar shots, shown as the dark green stripes at the bottom of Figure 1a, indicate that the lidar returns are not contiguous but are spaced by either 125 or $250 \mathrm{~m}$. The cloud profiling radar (CPR) footprints are 1.4-km wide across track and $2.5 \mathrm{~km}$ along track with a spacing of $1.4 \mathrm{~km}$ between profiles and will therefore be more contiguous than the higher resolution CALIOP data. They have a vertical resolution of $240 \mathrm{~m}$, while the lidar has a $30-\mathrm{m}$ resolution. Figure 2 shows the matched footprints in plan view. All 1-km MODIS pixels will be used instead of the nominal 2-km sampling indicated in the figure.

The discretized lidar sampling in Figure 1 requires interpolation to fill in the gaps. Interpolation is used whenever there is a gap no greater than the space in the lidar sampling to interpret the cloud boundaries. A schematic of the CPR sampling in Figure 1c shows that it misses most of the cirrus clouds but picks up the cloud extinction down to cloud base that is not seen by the lidar. The boundaries of the entire cloud

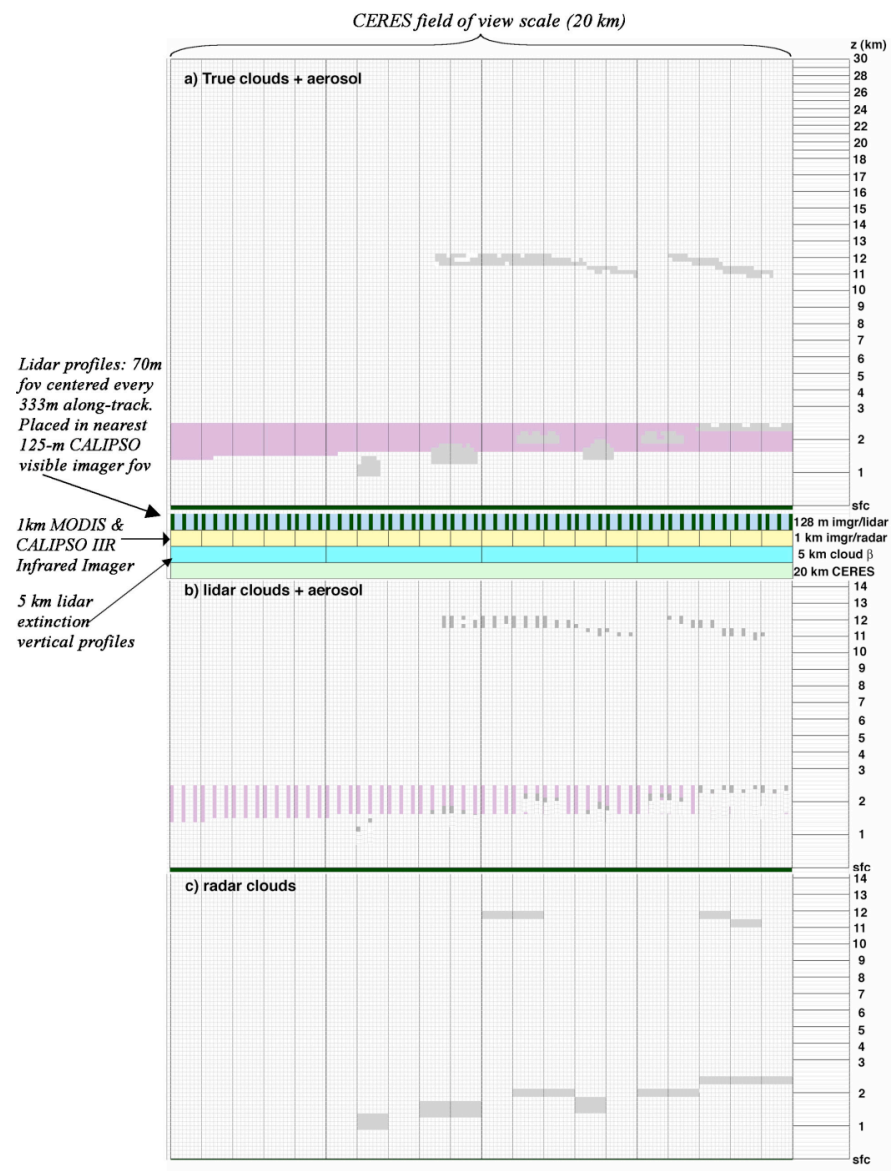

Figure 1. Schematic of a vertical profile aerosol and cloud layers for a) reference truth b) lidar sub-sampling with a $70 \mathrm{~m}$ fov profile every $333 \mathrm{~m}$ along the ground track, and c) radar with $\sim 1 \mathrm{~km}$ sampling along track.

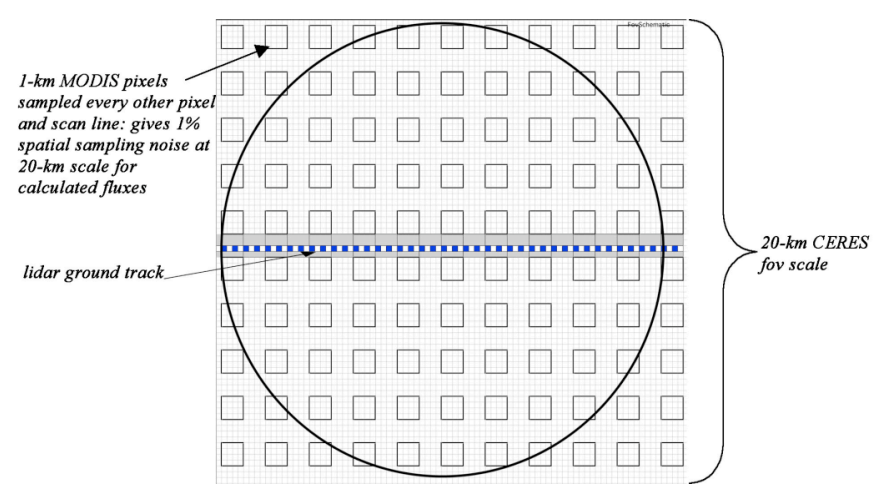

Figure 2. Schematic CERES FOV (circle) overlaid on sampled 1-km MODIS pixels (large squares, CPR track and IIR pixels (continuous gray), and CALIPSO lidar footprints (blue squares).

field are estimated by merging the interpolated lidar and CPR cloud boundaries. Differences in the vertical resolutions between the radar and lidar are reconciled by using the lidar cloud top and base altitudes for layers that are completely penetrated by the lidar beam and by using cloud top altitude only for optically thick clouds. The radar provides information about cloud base height for optically thick clouds. 


\section{Merged Data Products}

The goal of this effort is produce several products that can lead to effective understanding of cloud fields giving rise to the observed radiation, which can then be used to constrain radiative transfer calculations of surface and atmospheric heating rates based on the cloud properties.

The first step after matching the CALIOP and CPR data with the MODIS pixels is to determine how close the retrieved cloud properties are to those determined from the active sensors. The cloud top and base heights of the CERES-MODIS (CM) retrievals are then adjusted to match the active sensor results and the radiative properties recomputed as constrained by the MODIS data. These adjusted parameters for pixels along the CALIPSO track (gray area in Figure 2) will then be convolved into the CERES footprint without the other pixels off the track that fall within the CERES fov. A second dataset will convolve the unadjusted pixels along the CALIPSO track into the CERES fov. These two convolved datasets constitute track-sampled CERES single scanner footprints (SSF). The vertical profile information from the CALIOP-CPR data will also be used to adjust the CM cloud parameters off-track to provide a more realistic cloud field within the entire CERES footprint. These off-track pixel parameters will be convolved along with their along-track cousins to constitute a complete enhanced CERES SSF. The standard CERES SSF convolves the parameters from all of the pixels within the CERES fov using the standard CM retrievals. All convolutions weight the cloud properties for each MODIS pixel by the CERES pointspread function at that particular location relative to the CERES fov center. The result of these analysis and enhancement processes yields four datasets: standard and enhanced track-sampled SSF and standard and enhanced complete SSFs. From these results, the cloud fields will be used to compute the surface, atmospheric, and top-ofatmosphere radiative fluxes and divergences. The calculations are constrained by the CERES observations. The differences between the standard and enhanced products will be useful for determining the errors in the atmospheric heating rates. Differences in the fluxes between the enhanced track-sampled and complete SSFs yield the uncertainties in basing the assessment on the narrow fov's of the active instruments.

\section{RESUlts}

As of this writing, the CM pixels have been merged with the early releases of the CloudSat and CALIPSO retrievals. Figure 3 shows an example of the matched data for a track over the southeastern Indian Ocean during 6 August 2006. The RGB image at the top of the figure shows the cloud fields as observed by MODIS. The white area at left is located near Antarctica and is in darkness. The track proceeds northward to the right (latitude and longitude are shown at bottom of the figure). Generally CALIPSO yields slightly higher cloud tops (light blue areas) than CloudSat and picks up some boundarylayer clouds not observed by the radar. It also detects a boundary-layer aerosol layer (gold areas) between the clouds. It does not detect the depth of the deep storm cloud systems picked up by CloudSat (light green areas). The two instruments yield similar cloudy volumes (white regions) just below cloud top in many instances. The CM retrievals show water phase in red and ice phase in dark blue. The low-cloud heights from CM are typically too low, but the scattered clouds appear to be detected properly. In the deep storm systems, the CM retrieval determines the cloud phase as ice and cloud top is located at some point between the radar-only boundary and the cloud top.

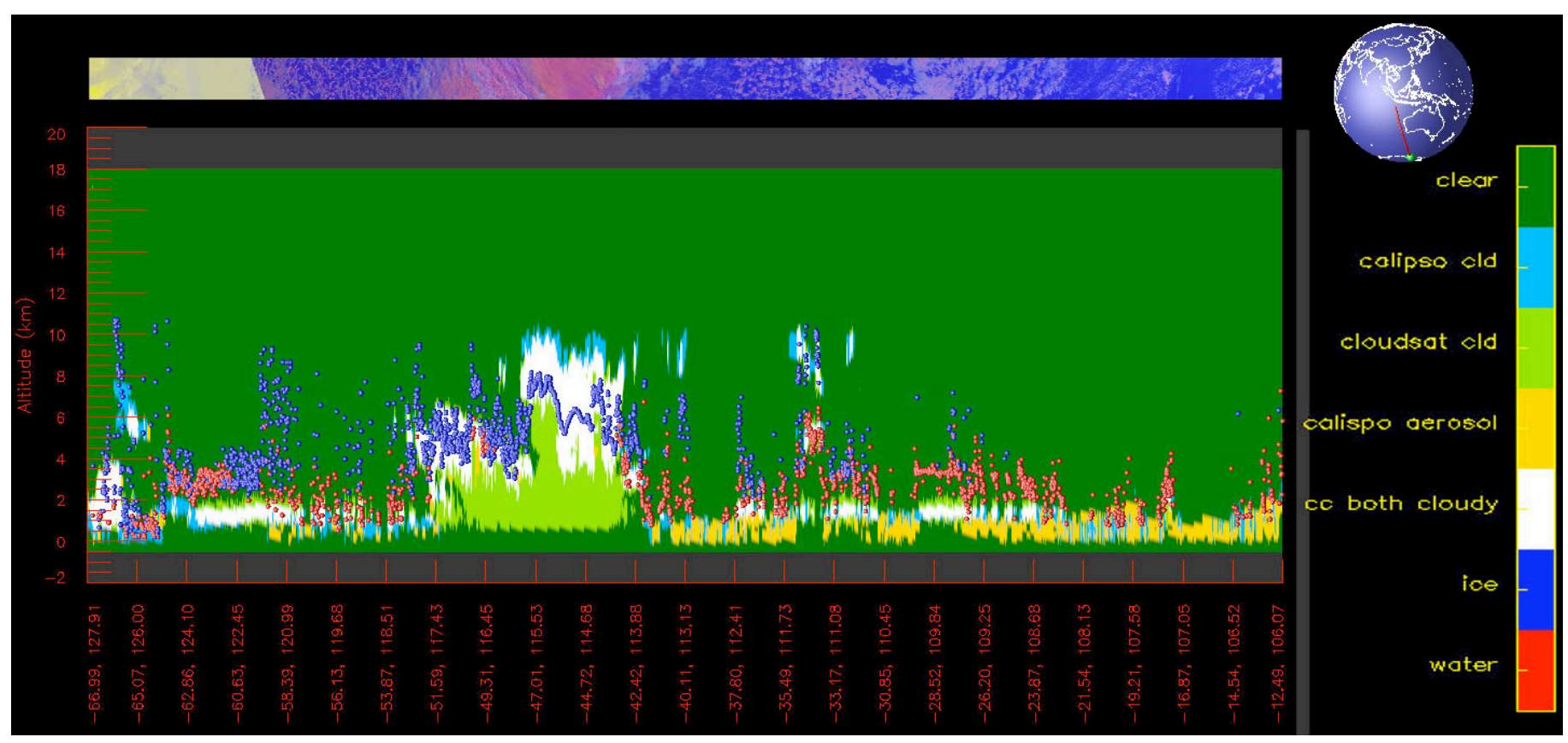

Figure 3. Example of matched CALIPSO, CloudSat, and CM cloud retirevals along the CALISPO ground track, 6 August 2006. Pseudoclor RGB image from MODIS data shown at top. Glround track for the image shown on globe at upper right. Each colored dot denotes a CM-retrieved cloud-top height. 
A discontinuity in the cloud heights is seen near the terminator. This "twilight" area causes more difficulty for the CM cloud mask than in full daylight or darkness. In some of the multilayered cloud cases, the CM result falls between the layers, while it corresponds to the highest cloud in other instances, probably reflecting the optical thickness of the higher cloud. Although the differences seen in this example are not uncommon, agreement between the $\mathrm{CM}$ and active sensor cloud heights are much better in many other marine areas.

\section{CONCLUDING REMARKS}

The next steps in the processing will use the information like that visualized in Figure 3 and adjust the CM retrievals so that the cloud boundaries and phase determinations are correct. That information will be used to improve the CM retrieval algorithms and to perform the radiative transfer calculations to produce heating profiles for the CERES footprints when the extinction profiles and higher level products become available form the CALISPO and CloudSat programs.

\section{ACKNOWLEDGMENT}

The authors are indebted to the members of the CALIPSO and CloudSat teams for providing the latest datasets for processing.

\section{REFERENCES}

[1] Wielicki, B. A., et al., "Clouds and the Earth's Radiant Energy System (CERES): Algorithm overview," IEEE Trans. Geosci. Remote Sens., vol. 36, pp. 1127-1141, 1998.

[2] Minnis, P., et al., "A multi-year data set of cloud properties derived for CERES from Aqua, Terra, and TRMM," Proc. 2006 IEEE Intl. Geosci. and Remote Sens. Symp., Denver, CO, 31 July - 4 Aug., CD-ROM, 02_50P03, 2006.

[3] Stephens, G. L., et al., "The CloudSat mission and the A-Train," Bull. Amer. Meteor. Soc., vol. 83, pp. 1771-1790, 2002.

[4] Winker, D. M., J. Pelon and M. P. McCormick, "The CALIPSO mission: Spaceborne lidar for observation of aerosols and clouds," Proc. SPIE Asia-Pacific Symposium on Remote Sensing of the Atmosphere, Environment and Space, Hangzhou, China, 23-27 October, 4893-01, 2002 . 